\title{
Proton pump inhibitor-induced exfoliative dermatitis: A case report
}

\author{
ZHIHONG QIU ${ }^{1}$, HONGTAO LIU ${ }^{1}$, LIEN HE ${ }^{1}$, YINLING MA ${ }^{1}$, \\ HAOJING SONG ${ }^{1}$, WANJUN BAI ${ }^{1}$ and MEILING YU ${ }^{2}$ \\ ${ }^{1}$ Department of Pharmacy, Hebei General Hospital, Shijiazhuang, Hebei 050051; \\ ${ }^{2}$ Department of Pharmacy, The First Affiliated Hospital of Bengbu Medical College, Bengbu, Anhui 233004, P.R. China
}

Received January 26, 2015; Accepted September 25, 2015

DOI: $10.3892 / \mathrm{etm} .2015 .2926$

\begin{abstract}
A 74-year-old female patient was admitted to hospital following a road accident with pains in the chest, abdomen, waist, back, nose, left wrist and lower limbs. After 1 week, the patient presented with gastrointestinal bleeding, and thus was treated with protein pump inhibitors (PPIs), including lansoprazole, esomeprazole and omeprazole enteric-coated tablets, in order to inhibit acid secretion and attenuate bleeding. However, the patient developed skin rashes on the chest and right lower limb and foot 28 days following treatment initiation. The skin rashes spread and ulcerated after 3 days, and were associated with tracheal mucosal injury and hemoptysis. Subsequently, treatment of the patient with PPIs was terminated, after which the tracheal hemoptysis and skin rashes markedly improved. In addition, no new skin rashes appeared following termination of the PPI treatment. In the present case, long-term treatment of an elderly patient with PPIs may have induced exfoliative dermatitis, due to hepatic ischemia, hypoxia and acute renal failure, which may have decreased the metabolism of PPIs, resulting in the accumulation of PPI metabolites.
\end{abstract}

\section{Introduction}

Exfoliative dermatitis, also known as erythroderma, is an uncommon but serious skin disorder which results in generalized eruption of the skin. It is usually caused by a variety of underlying dermatoses, drug reactions and malignancies, including lymphoma, leukemia and solid tumors (1-8). Previous studies have demonstrated that therapeutic agents including sulfonamides, captopril, phenobarbital, all-transretinoic acid and anti-tubercular drugs, such as rifampicin, isoniazid,

Correspondence to: Dr Meiling Yu, Department of Pharmacy, The First Affiliated Hospital of Bengbu Medical College, 287 Changhuai Road, Bengbu, Anhui 233004, P.R. China

E-mail: yumeiling409@sohu.com

Key words: proton pump inhibitors, exfoliative dermatitis ethambutol and pyrazinamide, may induce exfoliative dermatitis (3-8).

Protein pump inhibitors (PPIs) are commonly-used prescription drugs, which can inhibit gastric acid secretion by blocking the gastric $\mathrm{H}^{+} / \mathrm{K}^{+}$-ATPase that is necessary for the final step of this process. PPIs are widely administered for the treatment of numerous acid-associated diseases, including peptic ulcer, gastroesophageal reflux disease and Zollinger-Ellison syndrome $(9,10)$. In addition, PPIs have an important role in eradicating infections caused by Helicobacter pylori, controlling gastrointestinal bleeding and preventing nonsteroidal anti-inflammatory drug-induced gastric mucosal injuries. Furthermore, Ramirez et al (11) suggested that the long-term use of PPIs may be considered a major risk factor in the occurence of anaphylaxis with common PPI-induced anaphylactic symptoms including skin rashes, erythema and systemic pruritus, and rare symptoms including urticaria, herpes and skin allergic edema. In severe cases, acute diffuse epidermal necrolysis and anaphylactic shock have also been reported (12).

\section{Case report}

On September $29^{\text {th }}$ 2014, a 74-year-old female patient was admitted to Hubei General Hospital (Hubei, China) following a road accident with pains in the chest, abdomen, waist, back, nose, left wrist and lower limbs. A cranial computed tomography (CT) scan did not detect any abnormalities, whereas a nasal CT scan suggested a right nasal passage bone fracture and a chest CT scan detected numerous bilateral rib fractures, bilateral small pneumothoraces, traumatic wet lungs and compression fractures of the T12 and L1 vertebral bodies. Prior to the accident, the patient had been healthy, without a history of food or drug allergies.

A physical examination of the patient revealed the following: Body temperature, $36.5^{\circ} \mathrm{C}$; pulse rate, $100 \mathrm{bpm}$; respiratory rate, 21 breaths per minute; blood pressure, 100/60 mmHg; and body weight, $53 \mathrm{~kg}$. The patient had perirhinal bruises, minor bleeding and local tenderness. Rough breathing sounds and diffuse moist rates were detected for both lungs. No spinal deformities were detected; however, movement of the thoracic and lumbar vertebrae was markedly restricted. In addition, the lower thoracic and lumbar vertebrae exhibited tenderness and percussive pain, which radiated 

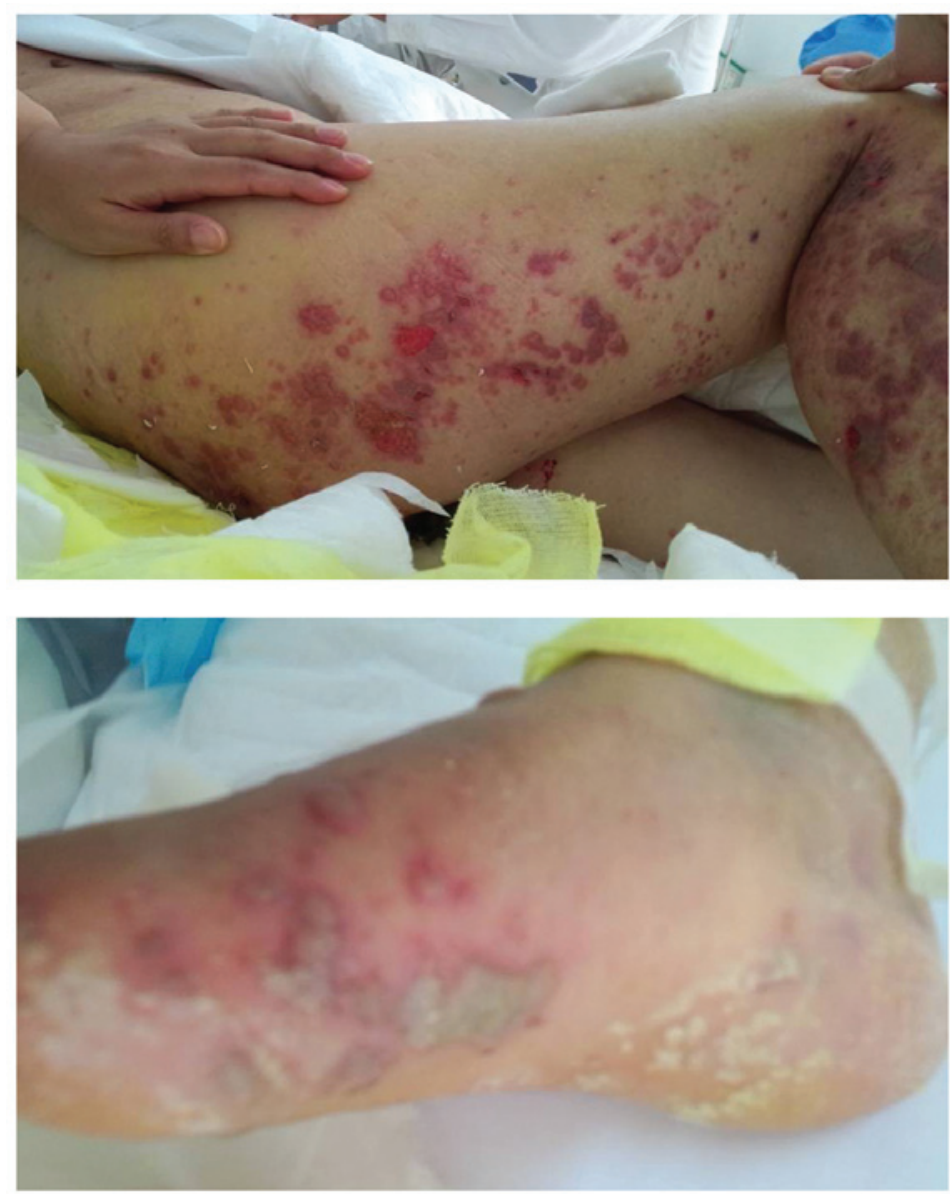

Figure 1. Skin rashes on the right lower limb and foot of an elderly female patient admitted following a road accident and undergoing long-term treatment with proton pump inhibitors. The rash on the right lower limb merged with that on the right foot.

to the lower limbs. The bilateral patellar tendon reflex and Achilles tendon reflex were significantly decreased. The lower limbs exhibited hypoesthesia, and the left side was particularly severe. Furthermore, the muscle strength of the lower limbs was significantly reduced, with grade 2 on the left iliopsoas, quadriceps femoris and extensor pollicis muscle, and grade $2+$ on the right iliopsoas, quadriceps femoris and extensor pollicis muscle. The muscle tension was normal and the dorsalis pedis artery pulse was palpated. The diagnosis at admission included: i) Chest and waist trauma, including compression fractures of the T12 and L1 vertebral bodies and incomplete paraplegia; ii) chest trauma, including multiple rib fractures, bilateral small pneumothoraces and traumatic wet lungs; iii) a nasal bone fracture; and iv) a left wrist trauma.

On October $5^{\text {th }}$, the patient was transferred from the Department of Orthopedics to the Department of Thoracic Surgery, and a daily intravenous administration of $30 \mathrm{mg}$ lansoprazole was initiated in order to prevent the development of a stress ulcer. On October $8^{\text {th }}$, the patient complained of abdominal pain, and a gastric tube was inserted for gastrointestinal decompression, after which blood was detected in the drained gastric fluid. Subsequently, the patient was diagnosed with gastrointestinal bleeding and was treated with the following hemostatic therapies, daily intravenous injections of 2 units hemocoagulase agkistrodon and 1,000 units thrombin lyophilized powder, administered via the nasal cavity every
4 h. A subsequent gastroscopy detected duodenal bulb bleeding, which was associated with the formation of a blood clot. As a result of the gastrointestinal bleeding, the once daily intravenous drip of $30 \mathrm{mg}$ lansoprazole was increased to twice daily.

On October $11^{\text {th }}$, the volume of gastric drainage significantly increased, and thus hemocoagulase agkistrodon and thrombin lyophilized powder injections were administered at the same doses as previously on October $8^{\text {th }}$, in order to attenuate the bleeding. In addition, the intravenous drip administering 30 mg lansoprazole was replaced with an $80 \mathrm{mg}$ esomeprazole intravenous drip twice daily, in order to inhibit acid secretion.

On October $13^{\text {th }}$, the gastrointestinal bleeding was controlled; however, the patient presented with paradoxical respiration, which progressively deteriorated. Subsequently, the patient was transferred to an operating room for emergency open reduction and internal fixation of numerous rib fractures and the sternal fracture. The surgery was successful; however, the patient remained critically ill, and thus was transferred to the intensive care unit (ICU) for continued treatment.

In the ICU, the patient once again suffered from gastrointestinal bleeding and subsequent acute kidney injury (AKI); therefore PPIs were administered in order to inhibit acid secretion. An intravenous drip administering $40 \mathrm{mg}$ esomeprazole sodium twice daily was administered from October $14^{\text {th }}$ to $15^{\text {th }}$ prior to continuous pumping of $200 \mathrm{mg}$ esomeprazole from 
October $15^{\text {th }}$ to $17^{\text {th }}$. This regimen was subsequently repeated; an intravenous drip administering $40 \mathrm{mg}$ esomeprazole sodium twice daily was administered from October $18^{\text {th }}$ to $26^{\text {th }}$ prior to continuous pumping of $200 \mathrm{mg}$ esomeprazole from October $26^{\text {th }}$ to $28^{\text {th }}$. On October $28^{\text {th }}$, the gastrointestinal bleeding was markedly attenuated and the continuous pumping of $200 \mathrm{mg}$ esomeprazole was replaced with once daily nasal administration of $40 \mathrm{mg}$ omeprazole enteric-coated capsules. The patient presented with systemic edema and a significant decrease in the volume of urine on October $19^{\text {th }}$; however both were markedly improved following treatment with hemocoagulase agkistrodon and thrombin lyophilized powder.

On the night of October $31^{\text {st }}$, minor skin rashes were detected on the chest, right lower limb and foot of the patient. In addition, the patient lost the ability to autonomously expectorate, due to the overproduction of sputum. Therefore, a tracheotomy was conducted, and the patient was transferred to the Department of Thoracic Surgery on November $1^{\text {st }}$, after which treatment with omeprazole was terminated and $30 \mathrm{mg}$ lansoprazole was intravenously administered once daily via a drip.

On November $3^{\text {rd }}$, the severity of the skin rashes on the chest and right lower limb and foot increased significantly, with the skin rash on the right lower limb merging with that on the right foot (Fig. 1). In addition, the routine blood examination revealed an elevated white blood cell count. On November $4^{\text {th }}$, the skin rashes ulcerated and tracheal hemoptysis occurred, after which a large volume of bloody mucinous sputum was suctioned off. Following a consultation between the Departments of Pharmacy, Dermatology and Nephrology, and the ICU, the patient was diagnosed with exfoliative dermatitis and treatment with lansoprazole was terminated. Anti-infection treatment, which comprised of intravenous administration of $4.5 \mathrm{~g}$ piperacillin sodium and tazobactam sodium three times a day and $200 \mathrm{mg}$ teicoplanin twice daily; and symptomatic treatment, in the form of daily intramuscular injection of $20 \mathrm{mg}$ diphenhydramine hydrochloride, were administered instead.

On November $5^{\text {th }}$, the sputum was yellow and white in appearance, and lacked traces of blood. On November $6^{\text {th }}$, the rashes on the chest and right lower limb and foot were markedly improved and the blisters were healed; however, the relatives of the patient subsequently decided to terminate all treatments and the patient succumbed to her traumas, gastrointestinal hemorrhage and infection-induced multiple organ (lung, heart and kidney) failure at 1:25 PM on November $10^{\text {th }}$. Written informed consent was obtained from the patient's family.

\section{Discussion}

The overuse of PPIs is a universal phenomenon in clinical practice (13-15), which may be due to a lack of clear and unified withdrawal instructions. Long-term treatment of a patient with a high-dose of PPIs has previously been associated with liver and renal dysfunction, gastrointestinal reactions, bone fractures, hypomagnesemia, malabsorption of vitamin C, B12 and iron, Clostridium difficile infections, and an increased incidence of pneumonia (16). In the present case, an elderly patient presented with bullous exfoliative dermatitis following long-term treatment with PPIs. To the best of our knowledge, this is the first report of PPI-induced exfoliative dermatitis.

Exfoliative dermatitis is a severe eruption of the skin associated with numerous drugs, including anti-tubercular drugs, such as rifampicin, isoniazid, ethambutol and pyrazinamide, sulfonamides, captopril, phenobarbital and all-transretinoic acid (3-8); however, to the best of our knowledge, PPI-induced exfoliative dermatitis has not previously been reported. The clinical symptoms of exfoliative dermatitis include: Systemic skin diffuse redness; desquamation (more severe on the face, hands and feet); blisters; erosion, exudation and scab formation on epidermal folds and mucosae; and pruritus and thermalgia, leading to systemic skin necrosis and generalized leaf-like desquamation. In addition, exfoliative dermatitis has previously been associated with severe systemic symptoms in some patients, including fever, shivering, and nausea and vomiting (1). In the case of patients with severe exfoliative dermatitis, a delay in the initiation of treatments is associated with life-threatening water and electrolyte disorders and the occurrence of secondary infections.

In the present case, an elderly female patient was treated with PPIs for 28 days (between October $6^{\text {th }}$ and November $2^{\text {nd }}$ ), and a skin rash was detected on October $31^{\text {st }}$, which markedly spread and ulcerated within 3 days of the initial presentation. Following a combined consultation between the Departments of Nephrology, Dermatology and Pharmacy, and the ICU, treatment of the patient with $30 \mathrm{mg}$ lansoprazole injection was terminated for 3 days, after which the skin rashes markedly improved, thus suggesting that a temporal relationship existed between the exfoliative dermatitis and PPI treatment. In addition, the patient exhibited hemoptysis as the skin rashes increased in severity; however, following termination of lansoprazole treatment and administration of hemostatic drugs, the hemoptysis was markedly attenuated and the volume of bloody sputum was significantly decreased. On November $5^{\text {th }}$, sputum extracted from the patient was yellow and white in appearance, indicating respiratory mucosal injuries in the patient. The symptoms of the patient were consistent with exfoliative dermatitis, and a temporal relationship between this skin eruption and treatment with PPIs was again indicated.

The authors of the present case hypothesize that the occurrence of exfoliative dermatitis in the patient was closely associated with an accumulation of PPIs. Throughout the duration of the treatment, lansoprazole and esomeprazole were the predominant PPIs used to treat the patient. Lansoprazole is metabolized by cytochrome P450 (CYP)-2C19 and CYP3A4 into 5-hydroxy lansoprazole and sulfonic lansoprazole, which are excreted with bile and via defecation $(17,18)$. Esomeprazole $(\sim 73 \%)$ is metabolized by CYP2C19 into hydroxy and demethylic metabolites, which lack the ability to inhibit acid secretion, and $27 \%$ is metabolized by CYP3A4 into esomeprazole sulfone, which is responsible for the acid-inhibiting activity of esomeprazole (19). As a result, $~ 80 \%$ inactive and $<1 \%$ active metabolites are excreted in bile, and the remainder in feces. Lansoprazole and esomeprazole share various traits: Firstly, the major metabolites of both (sulfonic lansoprazole and esomeprazole sulfone for lansoprazole and esomeprazole, respectively) are metabolized by CYP3A4 in the liver; and secondly, the metabolites of both drugs are excreted through bile rather than via the kidneys. Previous studies 
have suggested that renal failure does not affect the secretion of metabolites; however, AKI may significantly reduce the activity of CYP3A4 $(20,21)$. Therefore, in the present case, AKI may have significantly decreased the metabolism of lansoprazole and esomeprazole in the liver, leading to the accumulation of PPIs. In addition, the patient was hypoxic and ischemic following the severe traffic accident, which may have reduced the metabolism of lansoprazole and esomeprazole in the liver and obstructed the secretion of the metabolites, leading to their accumulation.

In the present case, the clinician did not respond to the small number of spot-like skin rashes on the patient on the night of October $31^{\text {st }}$, and the rashes rapidly progressed to a severe eruption within 3 days. Of the PPI-induced skin anaphylactic reactions, papula $(43.8 \%)$ is the most commonly observed (12). To the best of our knowledge, the present case is the first to report the occurrence of PPI-induced exfoliative dermatitis, and aims to draw attention to the risk of PPI-induced anaphylaxis during clinical practice, particularly in high-risk patients, including the elderly and those with liver and renal dysfunction, or a history of allergies. The present case suggests that treatment of a patient with PPI should be terminated upon detection of an adverse reaction, in order to prevent the occurrence of subsequent severe adverse reactions and events.

\section{References}

1. Sehgal VN, Srivastava G and Sardana K. Erythroderma/exfoliative dermatitis: A synopsis. Int J Dermatol 43: 39-47, 2004.

2. Rym BM, Mourad M, Bechir Z, Dalenda E, Faika C, Iadh AM and Amel BO: Erythroderma in adults: A report of 80 cases. Int J Dermatol 44: 731-5, 2005.

3. Dua R, Sindhwani G and Rawat J: Exfoliative dermatitis to all four first line oral anti-tubercular drugs. Indian J Tuberc 57: 53-6, 2010.

4. Jaisuresh K: Pyrazinamide-induced exfoliative dermatitis in a patient on hemodialysis: A rare complication. Case Rep Nephrol 2013: 387293, 2013.

5. Rabbat JC, Lin MY and Moy JN: Induction of tolerance in a patient with a history of exfoliative dermatitis to trimethoprim-sulfamethoxazole. Ann Allergy Asthma Immunol 109: 360-361, 2012.

6. O'Neill PG, Rajan N, Charlat ML and Bolli R: Captopril-related exfoliative dermatitis. Tex Med 85: 40-41, 1989.
7. Sawaishi Y, Komatsu K, Takeda O, Tazawa Y, Takahashi I, Hayasaka K and Takada G: A case of tubulo-interstitial nephritis with exfoliative dermatitis and hepatitis due to phenobarbital hypersensitivity. Eur J Pediatr 151: 69-72, 1992.

8. Ipek Y, Hulya D and Melih A: Disseminated exfoliative dermatitis associated with all-transretinoic Acid in the treatment of acute promyelocyticleukemia. Case Rep Med 2012: 236174, 2012.

9. Wedemeyer RS and Blume H: Pharmacokinetic drug interaction profiles of proton pump inhibitors: An update. Drug Saf 37: 201-211, 2014.

10. Watson C, Zhu L, Guan S, Machen TE and Forte JG: Reaction of proton pump inhibitors with model peptides results in novel products. J Pharmacol Sci 122: 213-22, 2013.

11. Ramírez E, Cabañas R, Laserna LS, Fiandor A, Tong H, Prior N, Calderón O, Medrano N, Bobolea I, Frías J and Quirce S: Proton pump inhibitors are associated with hypersensitivity reactions to drugs in hospitalized patients: A nested case-control in a retrospective cohort study. Clin Exp Allergy 43: 344-352, 2013.

12. Chang YS: Hypersensitivity reactions to proton pump inhibitors. Curr Opin Allergy Clin Immunol 12: 348-353, 2012.

13. Hood W, McJunkin B, Warnock A, Girme A, Smith N and Robinson B: Proton pump inhibitor prescribing and costs in a large outpatient clinic. W V Med J 110: 16-21, 2014.

14. Mouterde O, Bellaïche M, Dumant C and Mallet E: Gastroesophageal reflux and proton pump inhibitors: Panacea or prescription abuse? Arch Pediatr 17: 739-740, 2010 (In French).

15. Akram F, Huang Y, Lim V, Huggan PJ and Merchant RA: Proton pump inhibitors: Are we still prescribing them without valid indications? Australas Med J 7: 465-470, 2014.

16. Reimer C and Bytzer P: Adverse events associated with long-term use of proton pump inhibitors. Ugeskr Laeger 174: 2289-2293, 2012 (In Danish).

17. Zhang D, Zhang Y, Liu M, Wang X, Yang M, Han J and Liu H: Pharmacokinetics of lansoprazole and its main metabolites after single and multiple intravenous doses in healthy Chinese subjects. Eur J Drug Metab Pharmacokinet 38: 209-215, 2013.

18. Andersson T: Pharmacokinetics, metabolism and interactions of acid pump inhibitors. Focus on omeprazole, lansoprazole and pantoprazole. Clin Pharmacokinet 31: 9-28, 1996.

19. Zvyaga T, Chang SY, Chen C, Yang Z, Vuppugalla R, Hurley J, Thorndike D, Wagner A, Chimalakonda A and Rodrigues AD: Evaluation of six proton pump inhibitors as inhibitors of various human cytochromes P450: Focus on cytochrome P450 2C19. Drug Metab Dispos 40: 1698-1711, 2012.

20. Kirwan CJ, MacPhee IA, Lee T, Holt DW and Philips BJ: Acute kidney injury reduces the hepatic metabolism of midazolam in critically ill patients. Intensive Care Med 38:76-84, 2012.

21. Philips BJ, Lane K, Dixon J and Macphee I: The effects of acute renal failure on drug metabolism. Expert Opin Drug Metab Toxicol 10: 11-23, 2014. 